\title{
Structural Studies and Protein Engineering of Inositol Phosphate Multikinase*[s]
}

Received for publication, April 9, 2012, and in revised form, August 3, 2012 Published, JBC Papers in Press, August 15, 2012, DOI 10.1074/jbc.M112.365031

\author{
Stuart Endo-Streeter, Man-Kin Marco Tsui, Audrey R. Odom ', Jeremy Block, and John D. York² \\ From the Departments of Pharmacology and Cancer Biology, and of Biochemistry, Howard Hughes Medical Institute, Duke \\ University Medical Center, Durham, North Carolina 27710
}

\begin{abstract}
Background: IPMK is a key enzyme in signaling required for cellular adaptation and organismal development.
Results: We report the structure of IPMK, use structure-based enzyme design and perform complementation analysis in model systems.

Conclusion: We define a basis for substrate selectivity and report that 6-kinase activity is important for signaling. Significance: Determination of the IPMK structure enabled rewiring of signaling in organisms.
\end{abstract}

Inositol phosphates (IPs) regulate vital processes in eukaryotes, and their production downstream of phospholipase $\mathrm{C}$ activation is controlled through a network of evolutionarily conserved kinases and phosphatases. Inositol phosphate multikinase (IPMK, also called Ipk2 and Arg82) accounts for phosphorylation of $\mathrm{IP}_{3}$ to $\mathrm{IP}_{5}$, as well as production of several other IP molecules. Here, we report the structure of Arabidopsis thaliana IPMK $\alpha$ at $2.9 \AA$ and find it is similar to the yeast homolog Ipk2, despite $17 \%$ sequence identity, as well as the active site architecture of human $\mathrm{IP}_{3} 3$-kinase. Structural comparison and substrate modeling were used to identify a putative basis for IPMK selectivity. To test this model, we re-engineered binding site residues predicted to have restricted substrate specificity. Using steady-state kinetics and in vivo metabolic labeling studies in modified yeast strains, we observed that K117W and K117W:K121W mutants exhibited nearly normal 6-kinase function but harbored significantly reduced 3-kinase activity. These mutants complemented conditional nutritional growth defects observed in ipmk null yeast and, remarkably, suppressed lethality observed in ipmk null flies. Our data are consistent with the hypothesis that IPMK 6-kinase activity and production of Ins $(1,4,5,6) \mathrm{P}_{4}$ are critical for cellular signaling. Overall, our studies provide new insights into the structure and function of IPMK and utilize a synthetic biological approach to redesign inositol phosphate signaling pathways.

Inositol phosphates (IPs) ${ }^{3}$ are a large class of distinct chemical codes that regulate various intracellular processes. Classically, IPmediated signaling begins with the production of IPs in response

* This work was supported by the Howard Hughes Medical Institute and National Institutes of Health Grant RO1 HL-55672 (both to J. D. Y.).

5 This article contains supplemental Figs. S1-S3.

${ }^{1}$ Present address: Dept. of Pediatrics, WA University School of Medicine, St. Louis, MO 63110.

${ }^{2}$ To whom correspondence should be addressed: Dept. of Pharmacology and Cancer Biology, Duke University Medical Center, DUMC Box 3813, Durham, NC 27710. Tel.: 919-681-6414; Fax: 919-668-0991; E-mail: john.york@ vanderbilt.edu.

${ }^{3}$ The abbreviations used are: IP, inositol phosphate; $I P_{4}$, tetrakisphosphate; $\mathrm{IP}_{5}$, inositol pentakisphosphate; IPMK, inositol phosphate multikinase; FOM, figure of merit; SAD, single anomalous dispersion; CSM, complete synthetic medium. to cleavage of phosphoinositides by phosphatidylinositol-specific phospholipase C (reviewed in Ref. 1). Additional IP species such as inositol tetrakisphosphate $\left(\mathrm{IP}_{4}\right)$ and inositol pentakisphosphate $\left(\mathrm{IP}_{5}\right)$ are generated by subsequent series of phosphorylation and dephosphorylation (2-5). Multiple IP species have been linked to processes, including metabolic regulation and nutrient selection, stress responses, nuclear mRNA export, apoptosis, RNA editing, and organism development $(6,7)$.

Inositol phosphate multikinase (IPMK) is among the enzymes central to the production of IP species downstream of phospholipase $\mathrm{C}$ activation. For clarity, we use IPMK throughout this manuscript, as it most accurately reflects the "promiscuous" 6-/3-/5-kinase functions of the enzyme. As a historical note, IPMK was first designated ArgRIII (later called Arg82) based on a yeast genetic screen designed to identify the components of a nutrient response pathway in which arginine served as the sole nitrogen source $(8-11)$. ArgRIII was then renamed Ipk2 based on the discovery that it functioned as an inositol phosphate kinase $(12,13)$, and this nomenclature was also used in referring to the plant gene orthologs when they were first identified and characterized as $A t \mathrm{Ipk} 2 \alpha$ and $A t \mathrm{Ipk} 2 \beta$ in $A$ rabidopsis $(14,15)$. IPMK has now been cloned from a variety of species and has been shown to function as a 6/3/5-kinase that catalyzes the phosphorylation of at least seven different IP species. Its loss of function in a variety of organisms is critical for survival (reviewed in Refs. 5 and 6).

To gain insights into the basis of IPMK substrate promiscuity, we initiated a structural approach of the plant enzyme, which possesses three kinase activities capable of phosphorylating the D-6, D-3, and D-5 ring positions of a variety of IP substrates (14). We obtained Arabidopsis thaliana IPMK $\alpha$ crystals, complete data sets of native and seleno-methionine derivatives, and calculated an electron density map from experimental phases. Concomitant to our studies, the yeast IPMK crystal structure was published (16). From these studies and a number of subsequent biological and protein engineering experiments, we now report the crystal structure of $A$. thaliana IPMK $\alpha$ at $2.9 \AA$, a basis of IP substrate binding, the kinetic characterization of the wild-type enzyme and selective K117W and K121W mutants, and the yeast and fly IP profiles and phenotypes of the K121W and K117W/K121W mutants. Collectively, our data build upon and 
extend insights derived from the yeast crystal structure and, for the first time, provide a re-engineering of IPMK to be substrate-selective. We exploit these mutants to address important biological questions related to IPMK function, and our data are consistent with a role for 6-kinase activity in the regulation of cellular and organismal signaling.

\section{EXPERIMENTAL PROCEDURES}

Plasmid Constructs-Full-length and N-terminal truncated (residues $16-286 ; \Delta 16)$ A thaliana IPMK were amplified from the pGEX-KG construct (14) with the restriction sites NdeI or PsiI ( $\mathrm{N}$ terminus or $\Delta 16$ truncation) and BamHI ( $\mathrm{C}$ terminus) and cloned into the $6 \mathrm{xHis}$-tagged vector $\mathrm{pET}-15 \mathrm{~b}$. The 16-286 construct was designed based on alignment with the Saccharomyces cerevisiae IPMK sequence when crystals of full-length A. thaliana IPMK were found to be twinned. The $5^{\prime}$-sense fulllength primer was 5' -CAT AGT GGA CAT ATG CAG CTCAAA GTC CCT GAA CAT CAG-3' (restriction sites are underlined). The $\Delta 16-286$ was $5^{\prime}$-CAG TAT TAT AAA GAC GGG AAG CCT GGT CCT CTC-3'. The 3'-antisense primer was 5'-TAC GGA TCC TCA TCA CTA AGA ATC TGC AGA CTC ATC TGC-3'. The constructs were transformed into XL1-blue chemically competent cells and plasmids extracted from transformants and sequenced at the Duke University DNA sequencing facility.

Generation of Altered Specificity or Kinase-dead ConstructsPCR mutagenesis based on the Stratagene QuikChange kit was used to generate the mutants K100A, K117W, K121W, and K117W:K121W in full-length $A$. thaliana IPMK in pET-15b. Lys-100 is part of the canonical inositol kinase $\mathrm{P} x x x \mathrm{D} x \mathrm{~K} x \mathrm{G}$ motif, whereas Lys-117 and Lys-121 were identified from the A. thaliana IPMK structure as potential sites for generating IP substrate-selective mutants.

Expression and Purification of Recombinant A. thaliana IPMK-Constructs were transformed into BL21(DE3) (Invitrogen) cells and protein expression was induced with $1 \mathrm{~mm}$ isopropyl- $\beta$-D-thiogalactopyranoside at $30{ }^{\circ} \mathrm{C}$ for $3 \mathrm{~h}$ (seleno-methionine labeled) or overnight (native). Seleno-methionine labeling was performed by the methionine inhibition process (17), and complete incorporation was confirmed by mass spectrometry (supplemental Fig. S1). Cells were suspended in lysis buffer (50 mM Tris- $\mathrm{HCl}$ (pH 7.5), 500 mм KCl, 5 mм imidazole, 5 mм $\beta$-mercaptoethanol, and 1 tablet "Complete Mini" protease inhibitor (Roche Applied Science) per $10 \mathrm{~g}$ of cell pellet) and lysed by passing three times through an M110L microfluidizer (Microfluidics). The soluble fraction was loaded onto a nickelnitrilotriacetic acid Superflow column (Qiagen), washed, resuspended in one column volume of buffer, and digested with human thrombin (Hematologic Technologies). The resin was repacked and run over a nickel-nitrilotriacetic acid plus benzamidine-Sepharose 4 Fast Flow (GE Healthcare) column to remove any low-affinity proteins, and the thrombin and fractions were collected. Fractions with protein were concentrated and run over a Superdex 200 HiLoad 16/60 gel filtration column (Pharmacia). Final purity was confirmed by SDS-PAGE gels, concentrated to $10 \mathrm{mg} / \mathrm{ml}$, aliquoted, and stored at $-80^{\circ} \mathrm{C}$.

Crystallization and Data Collection-Native $\Delta 16$ AtIPK $2 \alpha$ crystallized in 2.0-2.15 $\mathrm{M}$ ammonium sulfate and $0.1 \mathrm{M}$ Tris$\mathrm{HCl}, \mathrm{pH} 8.5$, at $16-20 \mathrm{mg} / \mathrm{ml}$. Full-length SeMet $A t \mathrm{IPK} 2 \alpha$ crys- tallized in $2.10 \mathrm{M}$ ammonium sulfate and $0.1 \mathrm{M}$ Tris- $\mathrm{HCl}, \mathrm{pH} 7.0$, at $18 \mathrm{mg} / \mathrm{ml}$. All crystals were grown by hanging-drop vapor diffusion using Hampton trays. Crystals were cryo-protected by successive transfer into $30 \%$ glycerol and saturated ammonium sulfate at the crystallization $\mathrm{pH}$ in $5 \%$ glycerol increments. They were equilibrated overnight in the final protectant and then flash-frozen with liquid nitrogen. Data were collected at the Advanced Photon Source, Argonne National Laboratory (Argonne, IL), beamlines 22ID (SeMet) and 22BM (native) on MAR300 or MAR225 CCD detectors, respectively. The data were processed with XDS (18).

Structure Determination and Model Refinement-The SHELXC/D/E suite (19) was used to solve the structure by single-wavelength anomalous dispersion. Experimental synthesis maps were calculated to $3.10 \AA$ for both enantiomers in space groups $P 3_{2} 21$ and $P 3_{1} 21$. Comparison clearly revealed the correct hand and space group $\left(P 3_{2} 21\right)$. The initial phases were improved by solvent flattening and density modification with CNS (version 1.1) (20) using the $2.9 \AA \Delta 16$ AtIPK $2 \alpha$ native amplitudes. A polyalanine search model was generated in PyMOL (21) using the structures of S. cerevisiae IPMK and Homo sapiens IP3K (Protein Data Bank codes 2IEW, 1TZD, and 2AQX) (16, 22-24). Molrep (25) was used to fit the search model into the experimental density and provide a framework to build the remaining model. Model building was performed in iterative rounds using O (26), Coot (27), and KiNG (28). The initial poly-A model was refined by simulated annealing in CNS (20), and subsequent refinement rounds were carried out with Refmac5 (25). Translation-libration-screw refinement (29) was applied after completion of the backbone. Experimental density was observed equivalent to the $\beta 1$ strand of $S$. cerevisiae IPK2 and IP3K but could not be satisfactorily modeled. Structure validation was performed using MolProbity (30). Data and refinement statistics are reported in Table 1.

Design of Substrate-selective A. thaliana IPMK Mutants-To test the substrate selectivity motif and design substrate-selective mutants for in vivo signal probing, constructs were designed to block specific phosphate-binding pockets. The structures of A.thaliana IPMK, ADP-bound S. cerevisiae IPMK (Protein Data Bank code 2IF8), and substrate-bound H. sapiens IP3K (Protein Data Bank code 1W2C) were overlaid with LSQMAN (31), and the coordinates of $\operatorname{Ins}(1,4,5) \mathrm{P}_{3}$ from $H$. sapiens IP3K were transferred into the structure of $A$. thaliana IPMK. Using PyMOL (21), a model IP binding library was generated for $\mathrm{IP}_{6}$ (from the structure of ADAR2, Protein Data Bank code 1ZY7) in the 6-/3-/5-kinase reactions while conserving the original ring orientation. Phosphate groups were deleted as necessary to model the appropriate $\mathrm{IP}_{3}$ or $\mathrm{IP}_{4}$ species. Phosphate pockets were defined as the volume of space occupied by an equatorial phosphate group. Residues within $5 \AA$ of the putative $\gamma$ - or $\delta$-phosphate pockets were identified as potential candidates for mutation. Lys-117 and Lys-121 were mutated in silico using KiNG $(28,30)$ to residues able to occupy the corresponding phosphate pockets. Candidates were identified by selecting rotamer conformations without protein main-chain steric conflicts.

Substrate-selective Mutant Product Identification-Radiolabeled assays were performed to test the selectivity and identify the product of the mutant IPMKs. Ins $(1,4,5) \mathrm{P}_{3}$ was phosphory- 
lated by the $\mathrm{K} 121 \mathrm{~W}$ or $\mathrm{K} 117 \mathrm{~W} / \mathrm{K} 121 \mathrm{~W}$ mutants in the presence of $\left[\gamma_{-}{ }^{32} \mathrm{P}\right] \mathrm{ATP}$, and the products were separated via HPLC. Products were identified by treatment of $\left[{ }^{32} \mathrm{P}\right] \mathrm{IP}_{4}$ with 5 -ptase (selective for $\operatorname{Ins}(1,3,4,5) \mathrm{P}_{4}$ but not $\left.\operatorname{Ins}(1,4,5,6) \mathrm{P}_{4}\right)$ in the presence of $\left[{ }^{3} \mathrm{H}\right] \operatorname{Ins}(1,3,4,5) \mathrm{P}_{4}$ and separated with a $4.6 \times 125 \mathrm{~mm}$ Partisphere SAX anion exchange column over a $10 \mathrm{~mm}$ to $1.7 \mathrm{M}$ $\mathrm{NH}_{4} \mathrm{H}_{2} \mathrm{PO}_{4}$ gradient, whereas ${ }^{3} \mathrm{H}$ and ${ }^{32} \mathrm{P}$ activity was observed.

Generation and Purification of $\left[{ }^{32} \mathrm{P}\right] I \mathrm{P}_{4}$ Products $-\left[{ }^{32} \mathrm{P}\right]-$ $\operatorname{Ins}(1,4,5,6) \mathrm{P}_{4}$ and $\left[{ }^{32} \mathrm{P}\right] \operatorname{Ins}(1,3,4,5) \mathrm{P}_{4}$ were generated as follows. $10 \mu \mathrm{M} \operatorname{Ins}(1,4,5) \mathrm{P}_{3}$ and ATP with trace $\left[\gamma_{-}{ }^{32} \mathrm{P}\right] \mathrm{ATP}$ were treated with either K121W A. thaliana IPMK to generate $\operatorname{Ins}(1,4,5,6) \mathrm{P}_{4}$ or $D$. melanogaster IP3K to generate $\operatorname{Ins}(1,3,4,5) \mathrm{P}_{4}$. The reactions were performed in $50 \mathrm{~mm}$ Hepes, $\mathrm{pH}$ 6.6, $50 \mathrm{~mm} \mathrm{KCl}, 10 \mu \mathrm{M} \mathrm{ATP}, 1 \mathrm{~mm} \mathrm{MgCl}_{2}$, at $30{ }^{\circ} \mathrm{C}$ using $1 \mu \mathrm{g}$ of IPMK or IP3K and terminated by heat inactivation. $\left[{ }^{32} \mathrm{P}\right]-\mathrm{IP}_{4}$ products were purified over a $0.2 \mathrm{mM} \mathrm{HCl}$ to $0.5 \mathrm{M} \mathrm{HCl}, 2 \mathrm{mM}$ EDTA, 30-min gradient on a Partisphere SAX column, and $1-\mathrm{ml}$ fractions were collected. Fractions containing $\left[{ }^{32} \mathrm{P}\right] \mathrm{IP}_{4}$ were identified, and activity was quantitated by scintillation counting with ScintiSafe Econo2 (Fisher Chemicals) scintillation fluid in a Beckman LS6500 multipurpose scintillation counter. Samples were neutralized by the addition of $5 \mathrm{M} \mathrm{KOH,}$ until pH 7.0 was reached and brought to $50 \mathrm{~mm}$ Hepes, pH 7.0, aliquoted, and stored at $-20{ }^{\circ} \mathrm{C}$.

Inositol Phosphate Kinase Assays-All unlabeled IPs were purchased from Cell Signals, Inc. Tritiated IPs were purchased from American Radiolabeled Chemicals. Inositol phosphate steady-state kinetic reactions were performed in $50 \mathrm{~mm}$ Hepes, $\mathrm{pH}$ 6.6, $50 \mathrm{~mm} \mathrm{KCl}, 1.5 \mathrm{~mm}$ ATP, $5 \mathrm{~mm} \mathrm{MgCl}_{2}$, and $0.1 \mathrm{mg} / \mathrm{ml}$ bovine serum albumin at $30{ }^{\circ} \mathrm{C}$ and $9.8-1500 \mu \mathrm{M} \operatorname{Ins}(1,4,5) \mathrm{P}_{3}$, $\operatorname{Ins}(1,4,5,6) \mathrm{P}_{4}$, or $\operatorname{Ins}(1,3,4,5) \mathrm{P}_{4}$, with trace amounts of the matching species of $\left[{ }^{3} \mathrm{H}\right] \mathrm{IP}_{3}$ or $\left[{ }^{32} \mathrm{P}\right] \mathrm{IP}_{4}$. Reactions were halted with $2 \mu \mathrm{l}$ of $2.5 \mathrm{M} \mathrm{HCl}$ and separated by HPLC $\left({ }^{3} \mathrm{H}\right)$ or TLC $\left({ }^{32} \mathrm{P}\right) .{ }^{3} \mathrm{H}$ traces were quantitated with the Karat $32^{\mathrm{TM}}$ program, and ${ }^{32} \mathrm{P}$ TLC plates were quantitated with the ImageQuant ${ }^{\mathrm{TM}}$ program. All reactions were performed in at least triplicate, and data were analyzed with the Graphpad Prism ${ }^{\mathrm{TM}}$ program.

Yeast Strains and Media-Yeast strains $(12,14,32)$ were grown in complete minimal media with the appropriate amino acids and $2 \%$ sugar. Yeast lines were transformed using the lithium acetate method (33).

Yeast Construct Generation-Full-length A. thaliana IPMK was PCR-amplified from the pET-15 construct with primers for SalI and EcoRI N- and C-terminal sites. It was then ligated into pRS426 Cup1 and transformed into XL1-blue cells. An HA tag was included at the $C$ terminus. Plasmids were sequenced and point mutants were generated, as with the $\mathrm{pET}-15 \mathrm{~b}$ constructs.

Metabolic Labeling-Labeling and analysis were performed as described previously (34). Strains were inoculated from single colonies into $3 \mathrm{ml}$ of CSM-selective media, containing 20 $\mu \mathrm{Ci} / \mathrm{ml}\left[{ }^{3} \mathrm{H}\right] \mathrm{D}-m y o$-inositol and $50-150 \mu \mathrm{M} \mathrm{CuSO}_{4}$ and grown to saturation at $30^{\circ} \mathrm{C}$ and $250 \mathrm{rpm}$. The cultures were harvested by centrifugation, and soluble inositol phosphates were extracted. Organic and aqueous phases were separated by centrifugation, and soluble IPs in the aqueous phase were resolved by HPLC on a $4.6 \times 125 \mathrm{~mm}$ Partisphere SAX anion exchange column. Individual IP isomers were assigned on the basis of co-elution with IP standards.
Western Blots-Yeast extract blots were performed using mouse anti $\beta$-actin (mAbcam 8224) or $\alpha$-HA (primary) and goat anti-mouse (Odyssey no. 926-32210) and quantitated on an Odyssey Li-Cor thermal imager running software (version 2.1).

Plate Growth Assays-Single colonies were picked from streaks on selective media, suspended in sterile water, and water bath sonicated for $3 \times 5 \mathrm{~s} .5 \mu \mathrm{l}$ of 10 -fold serial dilutions were spotted on CSM-Ura+Dex, Glu-Ura+Dex, or OrnUra + Dex agar media and incubated at $30{ }^{\circ} \mathrm{C}$.

Generation of A. thaliana IPMK Transgenic D. melanogaster Lines-Wild-type and K121W A.thaliana IPMK in pET-15b were used as the templates to generate BamHI-XbaI fragments ligated into enhanced GFP and pUAST. The resultant p-element plasmids were injected into W1118 embryos, and the resultant transgenic lines were crossed with the $i p k 2^{20 \mathrm{~B}-3}$ allele on chromosome II.

Measurement of D. melanogaster Wing Morphology-Wings from 6-day-old adult males were dissected and mounted in glycerol. Pictures of the mounted wings were taken using a Nikon dissecting microscope and camera at $10 \times$ magnification. The relative sizes of the wings were measured by the Metamorph program.

\section{RESULTS}

Crystallization and Structure Determination-The structures of full-length and 16-286 $(\Delta 16) A$. thaliana IPMK were solved in space group $P 3_{2} 21$ by single wavelength anomalous dispersion at $3.1 \AA$, and the structure of $\Delta 16 \mathrm{~A}$. thaliana IPMK refined to $2.9 \AA$ with an $R / R_{\text {free }}$ of $23.64 / 24.61$. Each asymmetric unit contains two copies of $A$. thaliana IPMK, mirroring its elution as a dimer in gel chromatography (data not shown). 215 of 286 residues are modeled in each monomer, consisting of residues $41-70,81-175,183-230$, and 238-279. Anomalous diffraction density was observed for five of six seleno-methionine residues, the remaining residue in the disordered $\mathrm{N}$ terminus. $95.7 / 99.0 \%$ of the residues were in the favored/allowed regions of the Ramachandran plot. The solvent content was abnormally high at $76.5 \%$ with $V_{m}=5.22$ (35), but experimental maps reveal no density for additional molecules, and none can be modeled within the constraints of the asymmetric unit. Additional statistics are presented in Table 1.

Structural Description-A. thaliana IPMK has a similar secondary and tertiary structure to other members of the ATP grasp fold family $(36,37)$. It consists of eight $\alpha$-helices and 10 $\beta$-strands organized around a central $\beta$-sheet, backed by slightly off-parallel $\alpha$-helices organized into three domains (Fig. $1 A$ ). The $\mathrm{N}$ - and $\mathrm{C}$-terminal subdomains are $\alpha+\beta$ groupings, whereas the inositol binding domain consists of two $\alpha$-helices and connecting loop regions. To facilitate comparisons, we have followed the secondary structure assignment as used by Holms and Jogl (16) for the yeast structure (supplemental Fig. S2). The larger C-terminal subdomain makes up the majority of the structure of $A$. thaliana IPMK. Residues $94-102$ and 132277 are organized into five anti-parallel $\beta$-strands in a 3-2-1-4-5 order, flanked by helix $\alpha 6$ and backed by helices $\alpha 7, \alpha 8, \alpha 10$, and two smaller anti-parallel $\beta$-strands. Yeast helices $\alpha 5$ and $\alpha 9$ are not observed in the plant structure. $\alpha 5$ is part of an insert in 


\begin{tabular}{|c|c|c|}
\hline Data collection and phasing & $16-286$ & SeMet \\
\hline Space group & $P 3_{2} 21$ & $P 3_{2} 21$ \\
\hline Unit cell & $a=b=130.8 \AA, c=129.9 \AA ; \alpha=\beta=90^{\circ}, \gamma=120^{\circ}$ & $a=b=130.2 \AA, c=129.4 \AA ; \alpha=\beta=90^{\circ}, \gamma=120^{\circ}$ \\
\hline Wavelength $(\AA)$ & 1.000 & 0.97166 \\
\hline Resolution $(\AA ̊)$ & $46-2.9$ & $50-3.1$ \\
\hline Unique reflections & 28,738 & 44,459 \\
\hline Completeness (\%) & $100(100)^{a}$ & $100(100)$ \\
\hline$R_{\text {merge }} b$ & $8.5(52.4)$ & $9.2(52.6)$ \\
\hline FOM (SAD) & & 1.31 \\
\hline \multicolumn{3}{|l|}{ Refinement } \\
\hline Resolution range $(\AA)$ & $46-2.9(2.975-2.90)$ & \\
\hline No. of reflections & $314,752(43,621)$ & \\
\hline$R(\%)$ & $23.64(31.5)$ & \\
\hline$R_{\text {free }}(\%)$ & $24.61(37.0)$ & \\
\hline r.m.s.d. ${ }^{c}$ & & \\
\hline Bond length $(\AA)$ & 0.015 & \\
\hline Bond angle & $1.496^{\circ}$ & \\
\hline B factor $\left(\AA^{2}\right)$ main chain bonds & 0.917 & \\
\hline B factor $\left(\AA^{2}\right)$ side chain bonds & 2.725 & \\
\hline Protein atoms, number & 3444 & \\
\hline Ligand atoms, number & 30 & \\
\hline Residues in allowed $\varphi-\psi$ region & $99.5 \%$ & \\
\hline
\end{tabular}

the yeast sequence, whereas $\alpha 9$ is in the residues $231-237$ disordered region. Similarly, the $\beta 6$ strand in the same insert as helix $\alpha 5$ is not present. This may be an insert unique to $S$. cerevisiae IPMK as it is not present in $H$. sapiens IP3K, which has the same structure in this region as A. thaliana IPMK (Figs. 2 and $3 A)$. The inositol binding domain is inserted between strands $\beta 4$ and $\beta 5$. Helices $\alpha 6, \alpha 7$, and $\alpha 8$ connect $\beta 6$ and $\beta 7$, whereas the small sheet strands $\beta 8$ and $\beta 11$ are between $\alpha 8$ and $\beta 9$, and $\beta 10$ and $\alpha 10$, respectively. Packing between non-crystallographic copies is minimal, limited to parts of the $\mathrm{N}$-terminal domain (residues 54-58 and 68), with a few residues in the C-terminal domain (195-196, 203, 274, 277). Crystallographic packing is much more extensive, with contacts throughout the $\alpha$-helix-packed side of the central $\beta$-sheet in a back-to-back arrangement, in which half of the helices interact with one monomer and half with the other monomer. A second set of contacts exist at the edge of the central $\beta$-sheet, where the $\beta 6$ strand of chain A packs anti-parallel to the $\beta 6$ strand of the chain B symmetry mate. The two sets of symmetry contacts are on nearly orthogonal axes to each other; this may explain the ability of the $A$. thaliana IPMK crystals to grow to nearly $1-\mathrm{mm}$ dimensions despite their high (75\%) solvent content. Large solvent channels are evident when symmetry mates are generated but are not large enough to fit a third monomer.

The Structural Core of A. thaliana IPMK Is Homologous to Those of S. cerevisiae IPMK and H. sapiens IP3K-To examine the structural basis of substrate selectivity, the models of S. cerevisiae IPMK and H. sapiens IP3K were overlaid onto A. thaliana IPMK. As expected, the cores of each protein were nearly identical to one another (root mean square deviation $=$ 1.067 and $1.118 \AA$, Fig. 1C). Similarly, the active sites of all three proteins were well conserved, with the majority of conserved residues in either the central $\beta$-sheet or secondary structure elements that support it. The structure of the IP-binding domain was highly conserved between both IPMK species and diverges from $H$. sapiens IP3K, with the additional IP-binding domain inserts (Fig. 1C), which provide IP3K with its greater substrate selectivity $(23,24)$. Outside of the conserved core, there were several differences, concentrated in the external loops and species-specific inserts, and in the expanded inositol binding domain of IP3K.

Comparison of All Known Substrate and Non-substrate IP Species Suggests a Steric-based Binding Motif-To identify an IP substrate binding motif, we compared all known substrate and non-substrate IP species based on a common ring orientation and target hydroxyl position. Two phosphate groups were conserved in all substrate species and were identified as those with the most contacts with the IP3K substrate-bound structure (4' and $5^{\prime}$-phosphates). Orientated with the target hydroxyl pointed down and away from the plane of view, these phosphates are the first two located clock-wise from the target hydroxyl. No IP species with a $2^{\prime}$-phosphate group that was adjacent to the target hydroxyl and pointed in the same direction relative to the plane of the inositol ring was a substrate. A phosphate was also present for all substrates except Ins $(4,5) \mathrm{P}_{2}$ at one of the first two positions immediately counter-clockwise from the target hydroxyl. Three $\mathrm{IP}_{4}$ substrates of $A$. thaliana IPMK and a non-substrate species are shown in Fig. $2 A$, the common motif in Fig. $2 B$. The presence of a phosphate opposite the target hydroxyl does not appear to play any role and was predicted to be solvent-exposed or interact with residues able to adopt accommodating conformations such as lysine. During the course of this work, Chang and Majerus (38) independently proposed a similar theory for $H$. sapiens IPMK. Their model addresses the 5-/3-kinase activities of human IPMK and does not include a role for the 2 ' -hydroxyl and does not account for the 6-kinase activity of the yeast or plant homologues, which is severely reduced in the human form.

Putative Binding Modes of $\operatorname{Ins}(1,4,5) P_{3}, \operatorname{Ins}(1,4,5,6) P_{4}$, $\operatorname{Ins}(1,3,4,5) P_{4}$, and $\operatorname{Ins}(1,3,4,6) P_{4}$-All known substrate and non-substrate IP species were modeled into the active site of A. thaliana IPMK, following the proposed motif. As expected, the $A$. thaliana IPMK structure was able to accommodate all substrate species, and no non-substrate species except 


\section{Redesigning IPMK}

Ins $(1,4) \mathrm{P}_{2}$ and Ins $(1,3,4) \mathrm{P}_{3}$. These species lacked one of the two phosphate groups conserved in all substrate species. As expected, the ring positions that did not appear to require or exclude phosphate groups proved to be either solvent-exposed or had potential favorable interactions with residues Lys-117, Lys-121, Lys-153, or Arg-156. All possessed highly flexible side
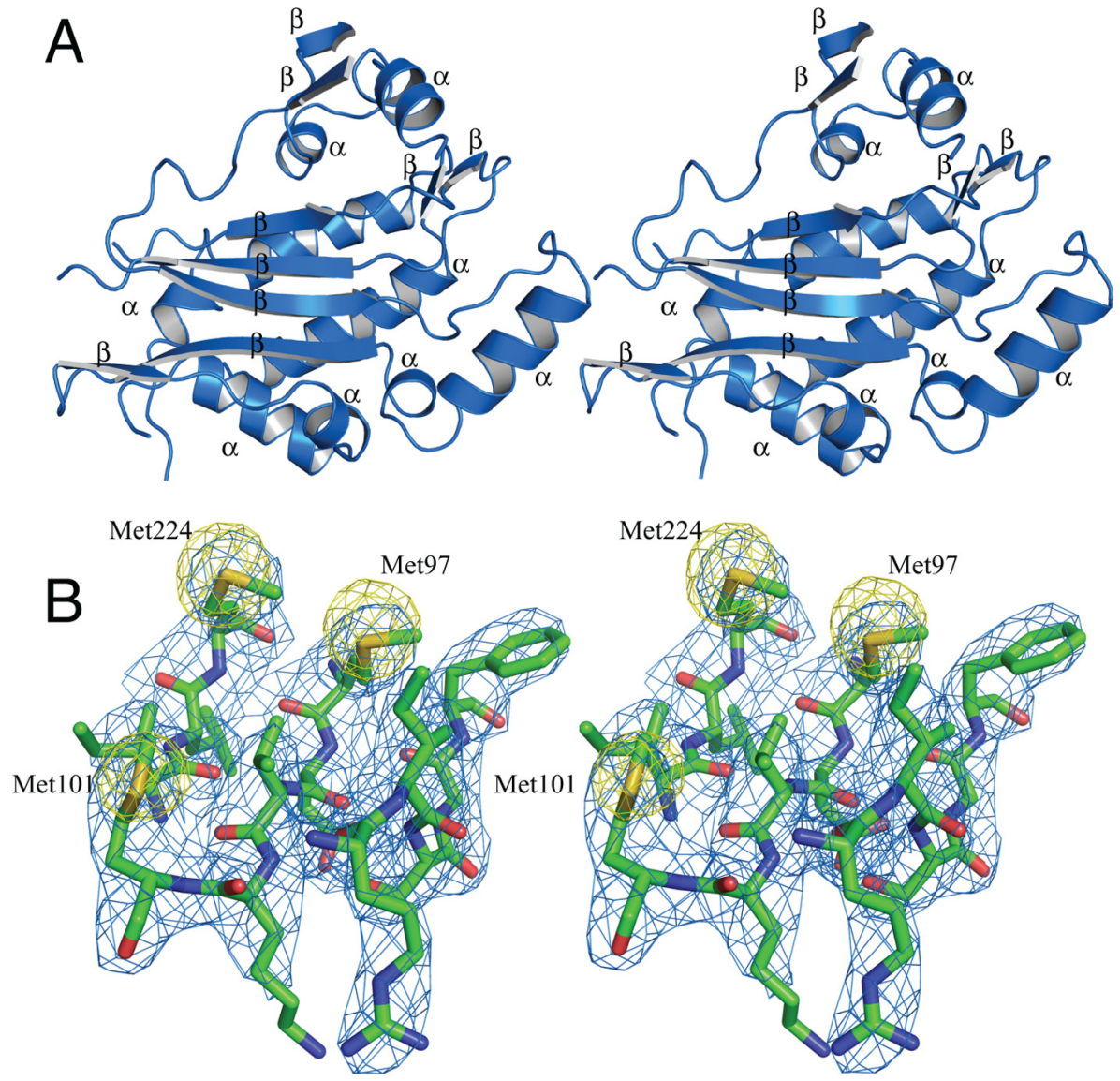

C
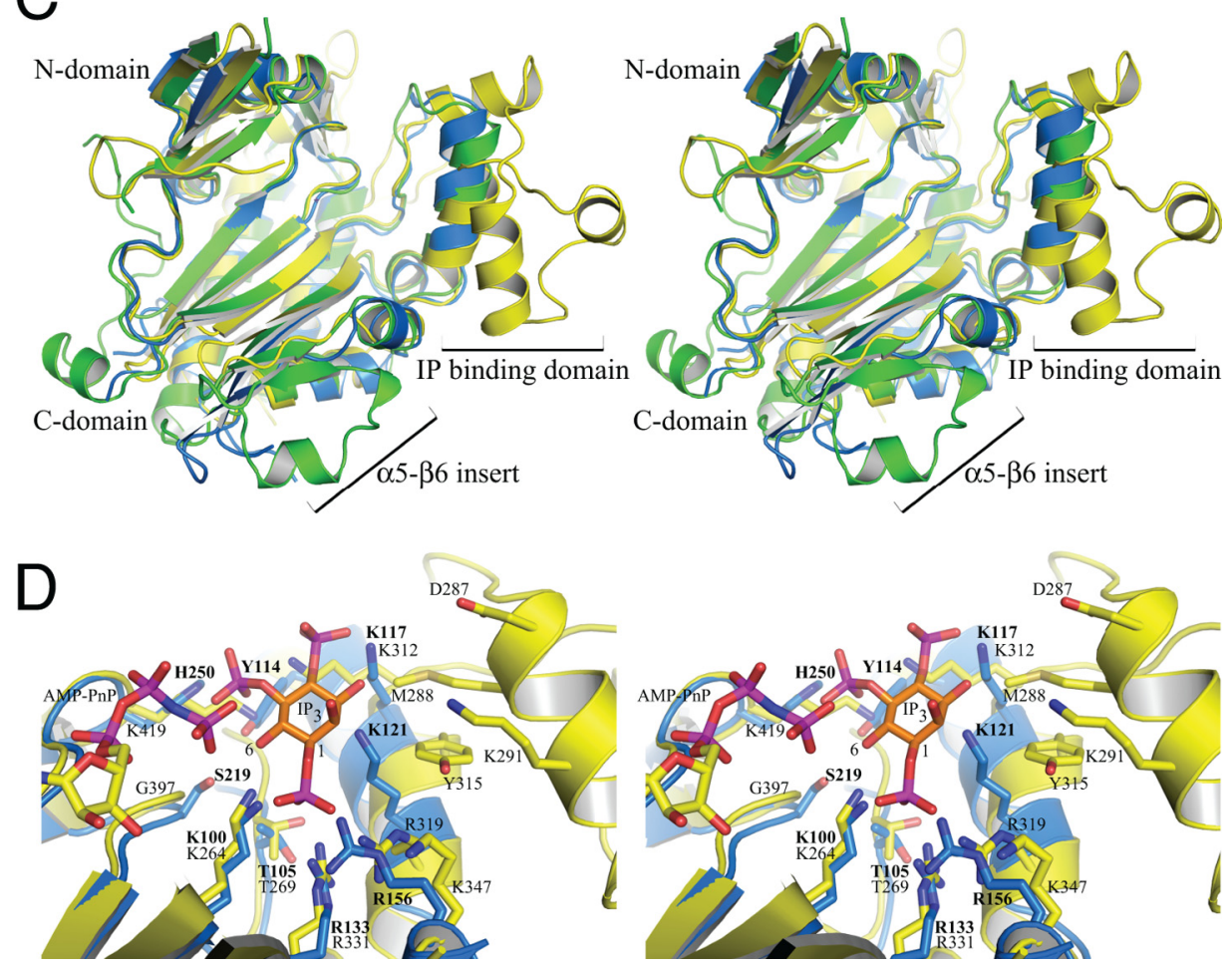


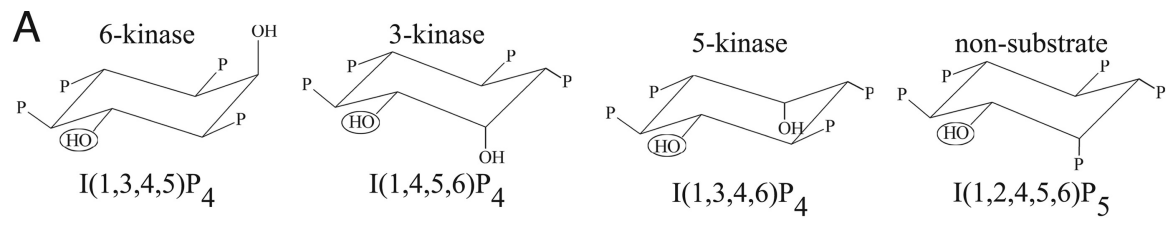

B

C

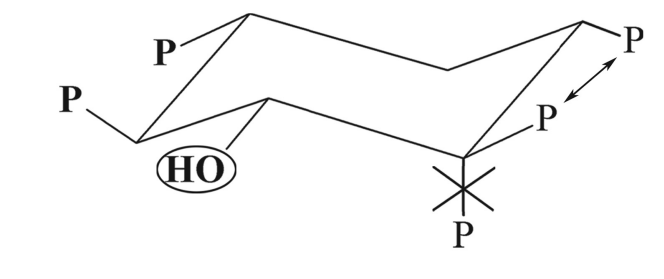

D
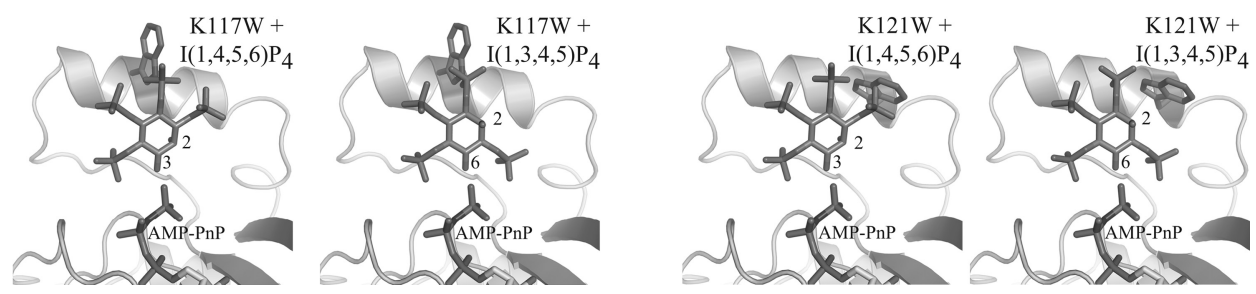

FIGURE 2. Inositol phosphate species, binding motif, and $\boldsymbol{A}$. thaliana IPMK selective designs. $A$, substrate and non-substrate IP species. Three $A$. thaliana IPMK IP substrate species, one for each kinase activity, and one non-substrate species are shown. All are orientated to preserve the position of the target hydroxyl (circled) and ring orientation. $B$, proposed IP substrate motif. Also shown are proposed rules for IP substrate selectivity by $A$. thaliana IPMK. Two phosphate groups are absolutely required (boldface type), the ring orientation must be maintained, one of two phosphate groups is required (arrows), and an axial phosphate cannot be tolerated if it occupies the first position clockwise of the target hydroxyl. C, phosphate binding pockets. Inositol in the active site of $A$. thaliana IPMK is shown conserving ring-carbon orientation. The phosphate pockets are designated $\alpha-\epsilon$ clockwise from the kinase target hydroxyl $\kappa$. Surrounding residues are omitted for clarity. $D$, proposed binding of $\mathrm{IP}_{4}$ species with K117W and K121W mutants. Ins $(1,3,4,5) \mathrm{P}_{4}$ and $\operatorname{lns}(1,4,5,6) \mathrm{P}_{4}$ are modeled with either $\mathrm{K} 117 \mathrm{~W}$ or K121W mutant models. $\mathrm{K} 117 \mathrm{~W}$ is predicted to have steric clashes with both $\mathrm{IP}_{4}$ species, whereas $\mathrm{K} 121 \mathrm{~W}$ is predicted to clash with only $\operatorname{lns}(1,4,5,6) \mathrm{P}_{4}$.

chains with minimal adjacent packing, presumably allowing the conformational flexibility required to tolerate the presence or absence of an IP phosphate group. IP species with a 2 '-phosphate group were modeled into this presumed active site and were found to have extensive steric clashes. Fig. $2 C$ shows inositol modeled in the proposed 6-kinase orientation in the active site of $A$. thaliana IPMK, with each phosphate pocket designated $\alpha-\epsilon$ located clockwise from the kinasetarget hydroxyl $\kappa$.

Substrate-selective Design and Product Identification-To test the substrate-selective motif, mutants were designed that were predicted to exclude specific classes of IP species from binding. Mutant A. thaliana IPMK constructs were treated with $\left[\gamma_{-}{ }^{32} \mathrm{P}\right] \mathrm{ATP}$ and $\operatorname{Ins}(1,4,5) \mathrm{P}_{3}$. The K117W mutant was predicted to partially inhibit both the 6 '-phosphorylation of $\operatorname{Ins}(1,3,4,5) \mathrm{P}_{4}$ and the $3^{\prime}$-phosphorylation of $\operatorname{Ins}(1,4,5,6) \mathrm{P}_{4}$ by blocking the $\gamma$-pocket, presumably occupied by a (different) phosphate for both $\mathrm{IP}_{4}$ species. Full inhibition was not expected due to the existence of a clash-relieving near-rotamer. K121W was predicted to severely inhibit the 3 '-phosphorylation of Ins $(1,4,5,6) \mathrm{P}_{4}$ but have little or lesser effect on the $6^{\prime}$-phos- phorylation of $\operatorname{Ins}(1,3,4,5) \mathrm{P}_{4}$, as the blocked $\delta$-pocket would not be occupied by a phosphate for the latter $\mathrm{IP}_{4}$ species (Fig. 2D). Treatment of $\operatorname{Ins}(1,4,5) \mathrm{P}_{3}$ with $\left[\gamma_{-}{ }^{32} \mathrm{P}\right] \mathrm{ATP}$ and K117W resulted in $\mathrm{IP}_{4}$ and $\mathrm{IP}_{5}$ formation, with reduced $\mathrm{IP}_{5}$ levels compared with wild-type, whereas $\mathrm{K} 121 \mathrm{~W}$ generated $\mathrm{IP}_{4}$ and no or trace $\mathrm{IP}_{5}$ (data not shown). Time courses of phosphorylation of $\left[{ }^{3} \mathrm{H}\right] \mathrm{IP}_{3}$ by wild-type and $\mathrm{K} 121 \mathrm{~W}$ confirmed the ${ }^{32} \mathrm{P}$-labeled observations (Fig. $3 A$ ). Treatment of the purified $\left[{ }^{32} \mathrm{P}\right] \mathrm{IP}_{4}$ product from K121W with 5-ptase identified it as $\operatorname{Ins}(1,4,5,6) \mathrm{P}_{4}$ (Fig. $3 B)$, as the internal control $\left[{ }^{3} \mathrm{H}\right] \operatorname{Ins}(1,3,4,5) \mathrm{P}_{4}$ was dephosphorylated to $\operatorname{Ins}(1,3,4) \mathrm{P}_{3}$; the $\left[{ }^{32} \mathrm{P}\right] \mathrm{IP}_{4}$ product was not and eluted at a different time point, matching the control $\left[{ }^{3} \mathrm{H}\right] \operatorname{Ins}(1,4,5,6) \mathrm{P}_{4}$ (data not shown).

K117W and K121W Mutants Possess Different Substrate Profiles-Michaelis-Menten steady-state kinetics experiments were performed to determine the changes in mutant specificity. The kinetic parameters $K_{m}$ and $V_{\max }$ were measured for the following reactions for wild-type IPMK and both selective mutants: $\operatorname{Ins}(1,4,5) \mathrm{P}_{3} \rightarrow \operatorname{Ins}(1,4,5,6) \mathrm{P}_{4}$ (6-kinase), Ins $(1,4,5$, 6) $\mathrm{P}_{4} \rightarrow \operatorname{Ins}(1,3,4,5,6) \mathrm{P}_{5}$ (3-kinase), and $\operatorname{Ins}(1,3,4,5) \mathrm{P}_{4} \rightarrow$ Ins $(1,3,4,5,6) \mathrm{P}_{5}$ (6-kinase) (Fig. $3 E$ and Table 2). The following

FIGURE 1. Structure and comparison of $A$. thaliana IPMK. $A$, stereo schematic of secondary structure. $B$, representative stereo density of core of $A$. thaliana IPMK and anomalous peaks. Single coefficient-weighted density (calculated by FFT in CCP4 4.6.1) is shown at $1.5 \sigma$ (blue), and anomalous density is shown at $12 \sigma$ around three of the five seleno-methionine residues used to solve the structure. C, stereo overlay of $A$. thaliana IPMK (blue), S. cerevisiae IPMK (green), and $H$. sapiens IP3K (yellow) structures. D, active site comparison of $A$. thaliana IPMK and $H$. sapiens IP3K with Ins $(1,4,5) P_{3}$. Stereo view of the active sites of $A$. thaliana IPMK and $\mathrm{H}$. sapiens IP3K with AMP-PnP from $\mathrm{H}$. sapiens IP3K and Ins $(1,4,5) \mathrm{P}_{3}$ modeled in the proposed 6-kinase orientation. Note the poor structural conservation on the opposite side of the inositol ring and much more extensive structure and contacts of $H$. sapiens IP3K. 
A

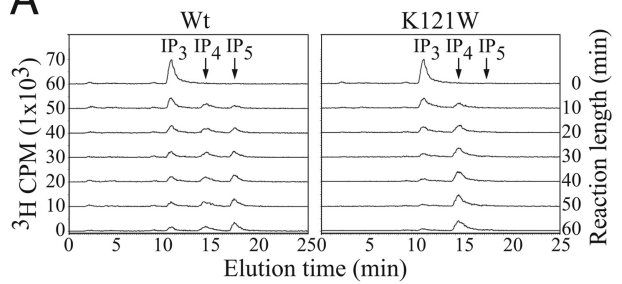

B

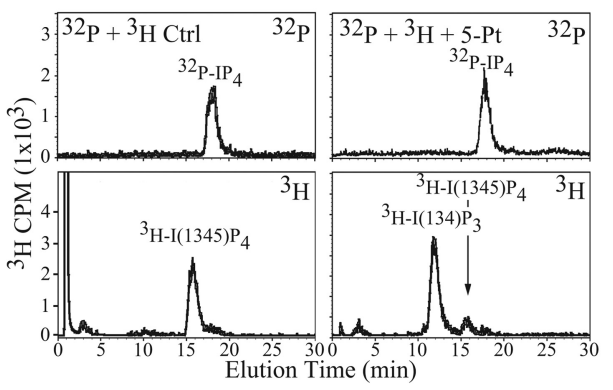

C
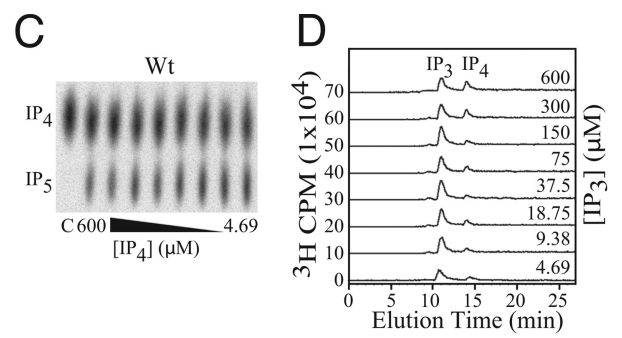

E

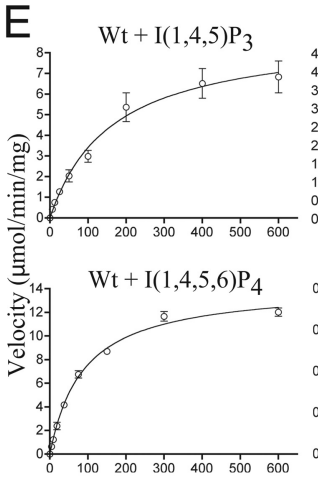

[IP substrate] $(\mu \mathrm{M})$
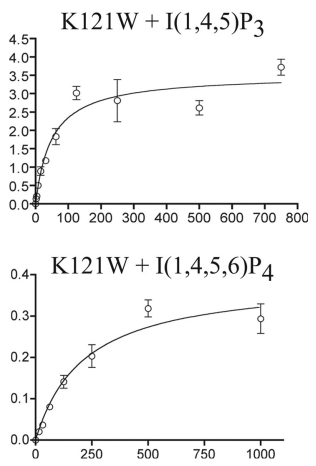

FIGURE 3. IP production, identity, and kinetics. $A$, production of $I P_{4}$ and $I P_{5}$ over time. Time courses of generation of $I P_{4}$ and $I P_{5}$ by wild-type and K121W A. thaliana IPMK from $\left[{ }^{3} \mathrm{H}\right] \ln s(1,4,5) \mathrm{P}_{3}$. Note the simultaneous appearance of $I P_{4}$ and $I P_{5}$ in the wild-type reactions, but no $I P_{5}$ signal for the $K 121 W$ reactions, even after 60 min. $B$, identification of the $\mathrm{K} 121 \mathrm{~W} \mathrm{IP}$ product as $\operatorname{lns}(1,4,5,6) \mathrm{P}_{4}$. Samples containing $\left[{ }^{32} \mathrm{P}\right] \mathrm{P}_{4}$ produced by $\mathrm{K} 121 \mathrm{~W}$ and $\left[{ }^{3} \mathrm{H}\right] \mathrm{Ins}(1,3,4,5) \mathrm{P}_{4}$ as an internal standard treated or not treated with 5 -ptas and separated by HPLC, and radioactivity was counted on ${ }^{3} \mathrm{H}$ and ${ }^{32} \mathrm{P}$ channels simultaneously. 5 -Ptase product is observed in only for $\left[{ }^{3} \mathrm{H}\right] I \mathrm{P}_{4}$. C, representative TLC plate of $\left[{ }^{32} \mathrm{P}\right] \mathrm{IP}$, assay. $D$, representative $\mathrm{HPLC}$ trace of $\left[{ }^{3} \mathrm{H}\right] \ln (1,4,5) \mathrm{P}_{3}$ assay. $\mathrm{E}_{1}$ Michaelis-Menten steady-state kinetic curves for $\operatorname{Ins}(1,4,5) \mathrm{P}_{3}$ and $\operatorname{Ins}(1,4,5,6) \mathrm{P}_{4}$ with wild-type and $\mathrm{K} 121 \mathrm{~W}$ A. thaliana IPMK.

TABLE 2

\section{Kinetics}

\begin{tabular}{|c|c|c|c|}
\hline IP Species & Wild-type & K117W & K121W \\
\hline $\begin{array}{l}\mathrm{I}(\mathbf{1}, \mathbf{4}, \mathbf{5}) \mathbf{P}_{3} \\
V_{\max }(\mu \mathrm{mol} / \mathrm{min} / \mathrm{mg}) \\
K_{m}(\mu \mathrm{M}) \\
k_{\text {cat }} / K_{m}\left(\mathrm{~s}^{-1} \mathrm{M}^{-1}\right)\end{array}$ & $\begin{array}{l}8.96 \\
162.2 \\
2.97 \times 10^{4} \\
\end{array}$ & $\mathrm{NP}^{a}$ & $\begin{array}{l}3.52 \\
50.05 \\
3.78 \times 10^{4}\end{array}$ \\
\hline $\begin{array}{l}\mathrm{I}(\mathbf{1}, \mathbf{3}, \mathbf{4}, \mathbf{5}) \mathbf{P}_{4} \\
V_{\max }(\mu \mathrm{mol} / \mathrm{min} / \mathrm{mg}) \\
K_{m}(\mu \mathrm{M}) \\
k_{\text {cat }} / K_{m}\left(\mathrm{~s}^{-1} \mathrm{M}^{-1}\right)\end{array}$ & $\begin{array}{l}6.06 \\
30.82 \\
1.06 \times 10^{5}\end{array}$ & $\begin{array}{l}6.11 \\
568 \\
5.79 \times 10^{3}\end{array}$ & $\begin{array}{l}6.61 \\
490 \\
7.27 \times 10^{3}\end{array}$ \\
\hline $\begin{array}{l}\mathbf{I}(\mathbf{1}, \mathbf{4}, \mathbf{5}, \mathbf{6}) \mathbf{P}_{\mathbf{4}} \\
V_{\max }(\mu \mathrm{mol} / \mathrm{min} / \mathrm{mg}) \\
K_{m}(\mu \mathrm{M}) \\
k_{\text {cat }} / K_{m}\left(\mathrm{~s}^{-1} \mathrm{M}^{-1}\right)\end{array}$ & $\begin{array}{l}11.04 \\
74.97 \\
7.91 \times 10^{4}\end{array}$ & $\begin{array}{l}0.96 \\
254 \\
2.04 \times 10^{3}\end{array}$ & $\begin{array}{l}0.39 \\
216 \\
9.73 \times 10^{2}\end{array}$ \\
\hline
\end{tabular}

${ }^{a} \mathrm{NP}$, not performed.

pattern in $k_{\text {cat }} / K_{m}$ was predicted based on the number of blocked sites for each combination of mutant and $\mathrm{IP}_{4}$ species, the presence of adjacent phosphate groups that would restrict the ability of the blocked phosphate to adopt a relieving conformation, and the possibility that alternate residue conformations existed to reduce clash: $\mathrm{WT} \geq \mathrm{K} 121 \mathrm{~W}+\mathrm{IP}_{3}>\mathrm{K} 117 \mathrm{~W}+$ $\operatorname{Ins}(1,3,4,5) \mathrm{P}_{4}>\mathrm{K} 121 \mathrm{~W}+\operatorname{Ins}(1,3,4,5) \mathrm{P}_{4}>\mathrm{K} 117 \mathrm{~W}+$ $\operatorname{Ins}(1,4,5,6) \mathrm{P}_{4}>\mathrm{K} 121 \mathrm{~W}+\operatorname{Ins}(1,4,5,6) \mathrm{P}_{4}$. This pattern was observed, supporting the inositol selectivity hypothesis. If loss of activity were due to a general inhibition of IP binding such as steric exclusion of the inositol ring, similar losses of activity would have been expected for all IP species for a given mutant. The conservation of the $1^{\prime}$ - and 4'-phosphates in two conserved pockets proposed by Ongusaha (39) and reviewed by Irvine (3) is not consistent with the single active site nor the pattern of activity loss.
In Vivo Different Inositol Phosphate Populations Are Altered by $K 121 \mathrm{~W}$ and $K 117 \mathrm{~W} / \mathrm{K} 121 \mathrm{~W}-$ To determine whether changes in the in vitro selectivity of the mutants affected in vivo IP production, the inositol profiles of $i p k 2 \Delta S$. cerevisiae with various $A$. thaliana IPMK constructs were measured by HPLC (Fig. 4A). In addition to the K121W and K117W/K121W double-mutants, empty vector, wild-type, and D247A kinase-dead constructs were also used. Similar levels of Ins, IP ${ }_{1}$, and IP 2 were measured for all constructs. $\mathrm{IP}_{3}$ levels were highest in the control and kinase-dead constructs, and progressively less in double mutant, K121W, and wild-type (negligible). Slightly elevated levels of $\mathrm{IP}_{3}$ observed in D247A mutant as compared with vector were not found to be statistically significant. $\mathrm{IP}_{4}$ levels were negligible in all constructs except the $\mathrm{K} 121 \mathrm{~W}$ and K117W/K121W mutants, approaching the control and kinasedead IP ${ }_{3}$ levels. IP Iproduced from IP $_{5}$ by IPK1(40)) levels were equivalent in $\mathrm{K} 121 \mathrm{~W}$ and wild-type but greatly reduced in the double mutant. The $\mathrm{IP}_{4}$ to $\mathrm{IP}_{6}$ ratios changed dramatically with the different selective mutants; although essentially, no $\mathrm{IP}_{4}$ is present with wild-type IPMK, levels of $\mathrm{IP}_{4}$ and $\mathrm{IP}_{6}$ are equivalent in $\mathrm{K} 121 \mathrm{~W}$, and $\mathrm{IP}_{4}$ is 6 -fold higher or greater than $\mathrm{IP}_{6}$ in K117W/K121W.

Mutants Have Different Rescue of Nitrogen Growth Defects Than Wild-type-Rescue of the ipmk $\Delta$ ornithine growth defect was compared between wild-type A. thaliana IPMK and the selective constructs (Fig. 4C). Yeast, plant, and fly IPMK have previously been shown to fully rescue growth with ornithine as the sole source of nitrogen $(12,14)$. Growth on CSM and glutamate media was unaffected by the deletion of IPMK but was lost when plated onto media containing ornithine as the sole nitrogen source. Both IPMK mutants were able to fully rescue 

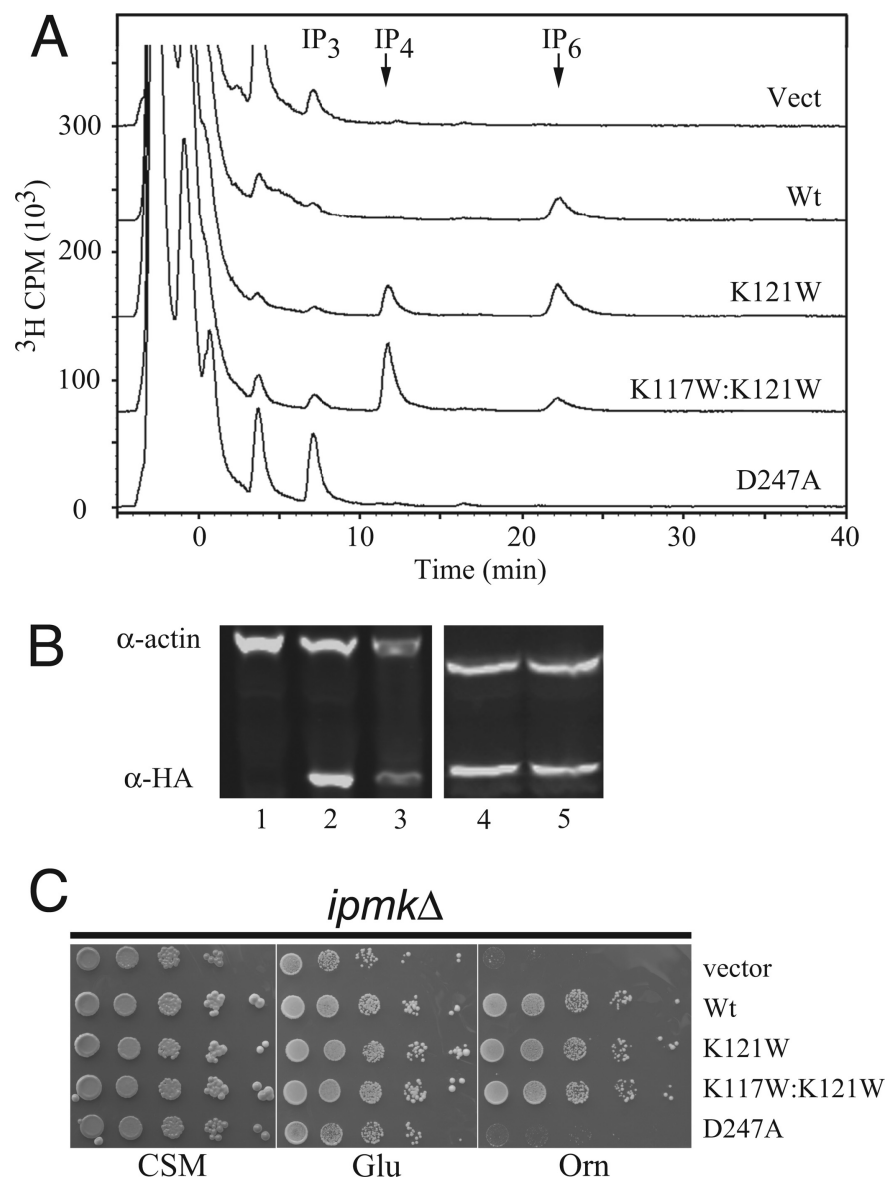

FIGURE 4. In vivo inositol phosphate production and rescue of ipmks. A, HPLC traces of soluble inositol phosphates. HPLC traces of soluble $\left[{ }^{3} \mathrm{H}\right] \mathrm{IP}$ species from ipmk $\Delta$ transformed with indicated $A$. thaliana IPMK constructs. $B$, Western blot of IPMK construct expression. Soluble $A$. thaliana IPMK expression detected with $\alpha$-HA and normalized to $\alpha$-actin. K117W/K121W and D247A blots are from a second series. C, growth on ornithine. 10-Fold serial dilution spot assays on CSM-Ura, Glu-Ura, or Orn-Ura media. Vect, vector.

growth on ornithine, with similar colony number and size as wild-type $A$. thaliana IPMK, whereas the catalytic knock-out D247A mutant failed to rescue.

Nitrogen Metabolism Control Does Not Appear to Require a Polyaspartate Region-It has been proposed that the polyaspartate region of S. cerevisiae IPMK, rather than the kinase functionality, is responsible for rescue of ornithine growth (41). Although kinase-dead mutants of yeast IPMK were reported to partially rescue growth on ornithine media, we observed that growth could also be rescued by plant and fly IPMK. Not only does $A$. thaliana IPMK clearly lack the polyaspartate insert or any sequence similarity to it (supplemental Fig. S3A), but the structure reveals that short of a complete unfolding of strands $\beta 9$ and $\beta 10$, there is no way a similar conformation could be adopted. Although residues 231-236 are disordered, any possible loop would be far too small to imitate the size of the 28-residue disordered region in yeast that includes the polyaspartate insert. Furthermore, the polyaspartate region in yeast begins pointed in the opposite direction of the beginning of the disordered region in plant (supplemental Fig. S3B). With only six residues, the plant disordered region would be unable to imitate even the residues of the yeast insert that are observable, much less the disordered region.
K121W Flies Have Rescued Growth but Exhibit Expressiondependent Defects in Wing Morphology-ipmks flies with transgenes for the K121W A. thaliana IPMK construct are able to mature, unlike ipmk $\Delta$ flies, which fail to progress beyond the larval stage. However, at lower expression levels, defects are observed in wing morphology, size, and posture (Fig. 5, $A-C$ ). Wing veins appear to be malformed, and the average wing size is reduced, whereas mature flies are unable to fully fold their wings along their back. At the L3 stage of development, wing discs are not observed for the K121W-6.1 transgene construct but are observed for the wild-type, wildtype plus K121W-6.1, and K121W-25.1 construct, which is a stronger promoter. Western blots confirm that expression of $\mathrm{K} 121 \mathrm{~W}$ is significantly higher in the K121W-25.1 construct (Fig. 5E).

\section{DISCUSSION}

Our work reveals the structure of plant IPMK, a basis of its substrate selectivity, and, through selective mutants, a linkage between specific IP species and phenotypes in yeast and flies. IPMK discriminates between the myriad different IP species through a combination of steric exclusion and requiring the occupation of specific phosphate binding pockets. It remains unclear whether or not there are accessory factors or posttranslational modifications that regulate IPMK 6-, 3-, or 5-kinase activities. However, recombinant protein produced in bacteria appears to perform phosphorylation of a multitude of substrates depending on their availability in kinetically similar fashion. With respect to proposed lipid kinase activity of mammalian IPMK, the structure of the plant enzyme and modeling of the active site suggests that 3-kinase activity toward phosphatidylinositol 4,5-bisphosphate may be prevented or greatly reduced by depth of the active site and steric incompatibilities. Indeed, we have been unsuccessful in measuring lipid kinase activity for recombinant $A$. thaliana IPMK in vitro or in vivo (data not shown).

The ability to alter the kinetic profiles of IPMK through single- and double-residue substitution confirms insights gleaned from substrate modeling. The restriction of the specificity of A. thaliana IPMK through mutations to both Lys-117 and Lys121 also allowed us to rewire inositol signaling pathways in two model systems. The combination mutant K117W/K121W achieved the best outcome and harbored normal $\operatorname{Ins}(1,4,5) \mathrm{P}_{3}$ 6-kinase, but greatly reduced $\operatorname{Ins}(1,4,5,6) \mathrm{P}_{4}$ 3-kinase activity. Our phenotypic studies in yeast indicate that the utilization of ornithine as a sole nitrogen source requires 6-kinase activity and that greatly reduced 3-kinase activity does not impair cellular responses.

Remarkably, we were also able to use a synthetic biology approach to probe inositol signaling specificity in the fruit fly D. melanogaster. Our recent work has defined an essential role for IPMK in fly development, proliferation, and cell death pathways. ${ }^{4}$ Interestingly, the low level expression of $\mathrm{K} 121 \mathrm{~W}$ A. thaliana IPMK mutant (promoter 6.1) is able to rescue the viability and imaginal disc development of ipmk null mutants;

\footnotetext{
${ }^{4}$ A. M. Seeds, M. M. Tsui, C. Sunu, E. P. Spana, and J. D. York, submitted for publication.
} 


\section{Redesigning IPMK}

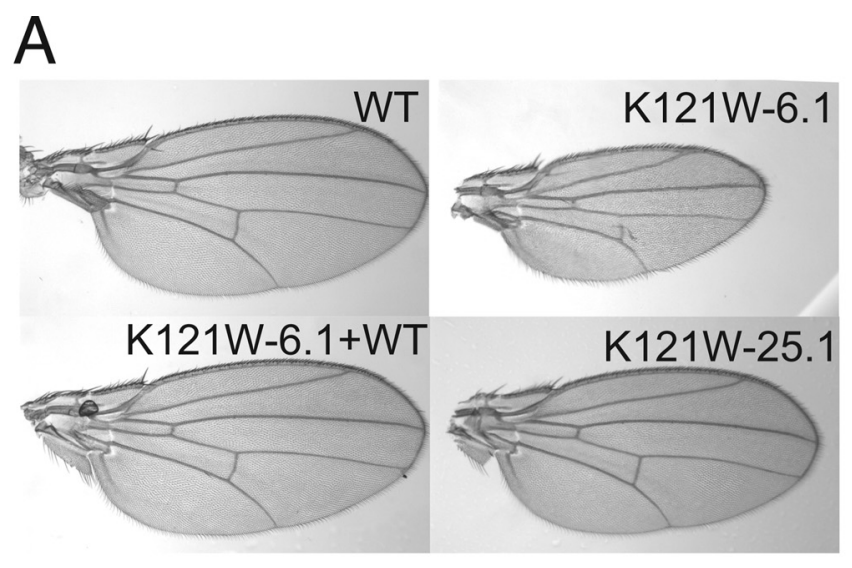

C

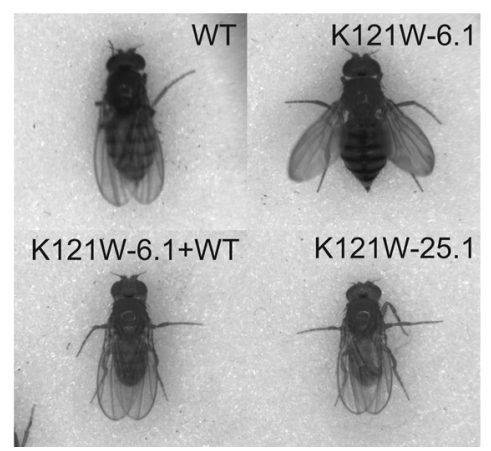

B

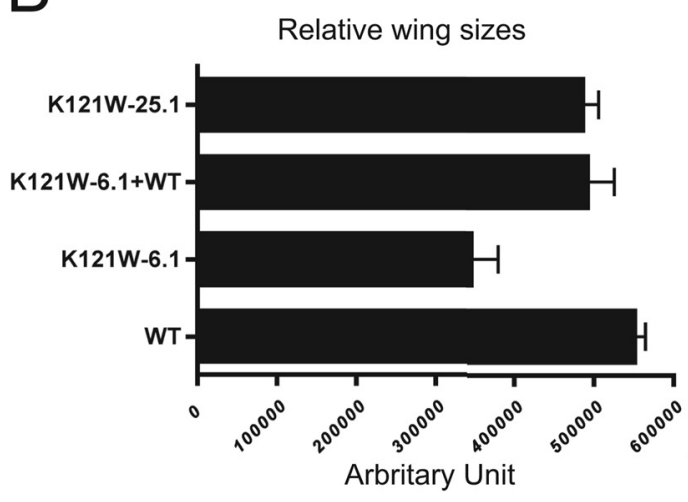

$\mathrm{E}$
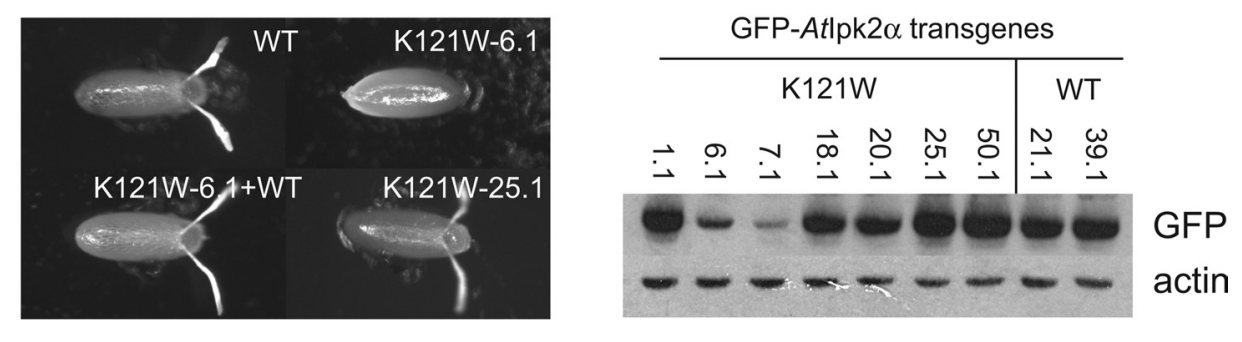

FIGURE 5. Fly complementation analysis and wing morphology. $A$ and $B$, representative wings and size distributions. Mean size \pm S.D., $n \geq 19$. $C$ and $D$, wing postures and eggs laid by indicated genotypes. WT, WT + K121W, ipmk $\Delta+\mathrm{K} 121 \mathrm{~W}-6.1$, and ipmk $\Delta+\mathrm{K} 121 \mathrm{~W}-25.1$. E, Western blot of GFPA. thaliana IPMK expression. Expression of various GFP-A. thaliana IPMK transgenes in D. melanogaster L3 larvae using $\alpha$-GFP and $\alpha$-actin as loading controls.

however, we noticed that proper wing patterning and position in adult flies remained perturbed, whereas high copy expression of the mutant (promoter 25.1) fully rescued viability and wing development. Our in vitro kinetics and yeast in vivo metabolic labeling of the $\mathrm{K} 121 \mathrm{~W}$ mutant indicates $\mathrm{IP}_{3} \rightarrow \mathrm{IP}_{4}$ production is normal, but conversion to $\mathrm{IP}_{5}$ is reduced but not abolished. We favor a model in which 6-kinase activity is critical; however, we cannot rule out that the residual 3-kinase, and possible untested 5-kinase, activities also contribute to the IMPK function in fly development. Further optimization of selective IPMK designs and testing of the K117W/K121W mutant in flies may help resolve these hypotheses in the fly system.

Our structure also provided suggestions that non-catalytic regions of IPMK may participate in cellular signaling and regulation. Of interest, the polyaspartate loop of S. cerevisiae IPMK, which was disordered in the crystal structure, was not present in A. thaliana IPMK. We also found that the loop of six residues between the $\beta 9$ and $\beta 10$ strands was disordered. Given that the plant protein does not harbor a polyaspartate region, the fact that the protein is fully capable of restoring growth with ornithine as the sole nitrogen source contradicts previous reports that the polyaspartate region is required for transcriptional responses mediated by IMPK (41). The fact that the rescue of growth by the plant enzyme depends on catalysis further emphasizes a role for IPMK and production of Ins $(1,4,5,6) \mathrm{P}_{4}$ in regulating transcriptional responses through the ArgR-Mcm 1 complex. We also note that recent reports of the structural basis for $\operatorname{Ins}(1,4,5,6) \mathrm{P}_{4}$ in binding to and regulating the histone deacetylase/co-repressor complex (HDAC3-NCOR2) provides an exciting new target for inositol signaling through IPMK (42).

Overall, our study provides insights into both the structure and biology of IPMK through a synthetic biology approach. Insights into substrate binding, kinase specificity and non-catalytic regions of IMPK have been used to answer unique biological questions in two model organisms, budding yeast and fruit flies. These have helped resolve relevance of signaling molecules and provide a foundation for future studies aimed at understanding signaling specificity and biology.

Acknowledgments - We thank the members of the York laboratory for helpful discussions, in particular Dr. D. Eric Dollins for discussions during phase determination, model building, and kinetic assays; Dr. Jim Otto and Peter Fridy for HPLC analysis, and Ace Hatch and Trang Pham for protocols and advice. We thank Drs. Jane and David Richardson and Dr. Bruce R. Donald (R01-GM-078031) for helpful support. We finally thank the $x$-ray crystallographic structure facility at Duke University and the Advanced Photon Source at Argonne National Laboratories and Advanced Light Source (Berkeley) where diffraction data were collected. 


\section{REFERENCES}

1. Drin, G., and Scarlata, S. (2007) Stimulation of phospholipase C $\beta$ by membrane interactions, interdomain movement, and $\mathrm{G}$ protein binding-how many ways can you activate an enzyme? Cell Signal. 19, 1383-1392

2. Gonzales, M. L., and Anderson, R. A. (2006) Nuclear phosphoinositide kinases and inositol phospholipids. J. Cell Biochem. 97, 252-260

3. Irvine, R. F. (2005) Inositide evolution-towards turtle domination? J. Physiol. 566, 295-300

4. Blazer-Yost, B. L., and Nofziger, C. (2005) Phosphoinositide lipid second messengers: New paradigms for transepithelial signal transduction. Pflugers Arch. 450, 75-82

5. Hatch, A. J., and York, J. D. (2010) SnapShot: Inositol phosphates. Cell 143, 1030-1030.e1

6. York, J. D. (2006) Regulation of nuclear processes by inositol polyphosphates. Biochim. Biophys. Acta 1761, 552-559

7. Seeds, A. M., Bastidas, R. J., and York, J. D. (2005) Molecular definition of a novel inositol polyphosphate metabolic pathway initiated by inositol 1,4,5-trisphosphate 3-kinase activity in Saccharomyces cerevisiae. J. Biol. Chem. 280, 27654-27661

8. Bechet, J., Greenson, M., and Wiame, J. M. (1970) Mutations affecting the repressibility of arginine biosynthetic enzymes in Saccharomyces cerevisiae. Eur. J. Biochem. 12, 31-39

9. Dubois, E., and Messenguy, F. (1994) Pleiotropic function of ArgRIIIp (Arg82p), one of the regulators of arginine metabolism in Saccharomyces cerevisiae. Role in expression of cell-type-specific genes. Mol. Gen. Genet. 243, 315-324

10. Messenguy, F., Dubois, E., and Boonchird, C. (1991) Determination of the DNA-binding sequences of ARGR proteins to arginine anabolic and catabolic promoters. Mol. Cell Biol. 11, 2852-2863

11. Dubois, E., and Messenguy, F. (1985) Isolation and characterization of the yeast ARGRII gene involved in regulating both anabolism and catabolism of arginine. Mol. Gen. Genet. 198, 283-289

12. Odom, A. R., Stahlberg, A., Wente, S. R., and York, J. D. (2000) A role for nuclear inositol 1,4,5-trisphosphate kinase in transcriptional control. Science 287, 2026-2029

13. York, J. D., Odom, A. R., Murphy, R., Ives, E. B., and Wente, S. R. (1999) A phospholipase C-dependent inositol polyphosphate kinase pathway required for efficient messenger RNA export. Science 285, 96-100

14. Stevenson-Paulik, J., Odom, A. R., and York, J. D. (2002) Molecular and biochemical characterization of two plant inositol polyphosphate 6-/3-/5kinases. J. Biol. Chem. 277, 42711-42718

15. Stevenson-Paulik, J., Bastidas, R. J., Chiou, S. T., Frye, R. A., and York, J. D. (2005) Generation of phytate-free seeds in Arabidopsis through disruption of inositol polyphosphate kinases. Proc. Natl. Acad. Sci. U.S.A. 102, 12612-12617

16. Holmes, W., and Jogl, G. (2006) Crystal structure of inositol phosphate multikinase 2 and implications for substrate specificity. J. Biol. Chem. 281, 38109-38116

17. Van Duyne, G. D., Standaert, R. F., Karplus, P. A., Schreiber, S. L., and Clardy, J. (1993) XDS atomic structures of the human immunophilin FKBP-12 complexes with FK506 and rapamycin. J. Mol. Biol. 229, $105-124$

18. Kabsch, W. (1993) XDS automatic processing of rotation diffraction data from crystals of initially unknown symmetry and cell constants. J. Appl. Cryst. 26, 795-800

19. Sheldrick, G. M. (1997) SHELX97 Manual, University of Gottingen, Germany

20. Brünger, A. T., Adams, P. D., Clore, G. M., DeLano, W. L., Gros, P., Grosse-Kunstleve, R. W., Jiang, J. S., Kuszewski, J., Nilges, M., Pannu, N. S., Read, R. J., Rice, L. M., Simonson, T., and Warren, G. L. (1998) Crystallography \& NMR system: A new software suite for macromolecular structure determination. Acta Crystallogr. D Biol. Crystallogr. 54, 905-921
21. DeLano, W. L. (2010) The PyMOL Molecular Graphics System, version 1.3r1, Schrödinger, LLC, New York

22. Chamberlain, P. P., Sandberg, M. L., Sauer, K., Cooke, M. P., Lesley, S. A., and Spraggon, G. (2005) Structural insights into enzyme regulation for inositol 1,4,5-trisphosphate 3-kinase B. Biochemistry 44, 14486-14493

23. Miller, G. J., and Hurley, J. H. (2004) Crystal structure of the catalytic core of inositol 1,4,5-trisphosphate 3-kinase. Mol. Cell 15, 703-711

24. González, B., Schell, M. J., Letcher, A. J., Veprintsev, D. B., Irvine, R. F., and Williams, R. L. (2004) Structure of a human inositol 1,4,5-trisphosphate 3-kinase: Substrate binding reveals why it is not a phosphoinositide 3-kinase. Mol. Cell 15, 689-701

25. Collaborative Computational Project, Number 4 (1994) The CCP4 suite: programs for protein crystallography. Acta Crystallogr. D Biol. Crystallogr. 50, 760-763

26. Jones, T. A., Zou, J. Y., Cowan, S. W., and Kjeldgaard. (1991) Improved methods for building protein models in electron density maps and the location of errors in these models. Acta Crystallogr. A 47, 110-119

27. Emsley, P., and Cowtan, K. (2004) Coot: Model-building tools for molecular graphics. Acta Crystallogr. D Biol. Crystallogr. 60, 2126-2132

28. Lovell, S. C., Davis, I. W., Arendall, W. B., 3rd, de Bakker, P. I., Word, J. M., Prisant, M. G., Richardson, J. S., and Richardson, D. C. (2003) Structure validation by $C \alpha$ geometry: $\phi, \psi$, and $C \beta$ deviation. Proteins $50,437-450$

29. Winn, M. D., Isupov, M. N., and Murshudov, G. N. (2001) Use of TLS parameters to model anisotropic displacements in macromolecular refinement. Acta Crystallogr. D Biol. Crystallogr. 57, 122-133

30. Davis, I. W., Murray, L. W., Richardson, J. S., and Richardson, D. C. (2004) MOLPROBITY: Structure validation and all-atom contact analysis for nucleic acids and their complexes. Nucleic Acids Res. 32, W615-619

31. Kleywegt, G. J. (1996) Use of non-crystallographic symmetry in protein structure refinement. Acta Crystallogr. D Biol. Crystallogr. 52, 842- 857

32. Seeds, A. M., Sandquist, J. C., Spana, E. P., and York, J. D. (2004) A molecular basis for inositol polyphosphate synthesis in Drosophila melanogaster. J. Biol. Chem. 279, 47222-47232

33. Ito, H., Fukuda, Y., Murata, K., and Kimura, A. (1983) Transformation of intact yeast cells treated with alkali cations. J. Bacteriol. 153, 163-168

34. Stolz, L. E., Huynh, C. V., Thorner, J., and York, J. D. (1998) Identification and characterization of an essential family of inositol polyphosphate 5 -phosphatases (INP51, INP52, and INP53 gene products) in the yeast Saccharomyces cerevisiae. Genetics 148, 1715-1729

35. Matthews, B. W. (1968) Solvent content of protein crystals. J. Mol. Biol. 33, 491-497

36. Murzin, A. G. (1996) Structural classification of proteins: New superfamilies. Curr. Opin. Struct. Biol. 6, 386-394

37. Fan, C., Moews, P. C., Shi, Y., Walsh, C. T., and Knox, J. R. (1995) A common fold for peptide synthetases cleaving ATP to ADP: Glutathione synthetase and D-alanine:D-alanine ligase of Escherichia coli. Proc. Natl. Acad. Sci. U.S.A. 92, 1172-1176

38. Chang, S. C., and Majerus, P. W. (2006) Inositol polyphosphate multikinase regulates inositol 1,4,5,6-tetrakisphosphate. Biochem. Biophys. Res. Commun. 339, 209-216

39. Ongusaha, P. P., Hughes, P. J., Davey, J., and Michell, R. H. (1998) Inositol hexakisphosphate in Schizosaccharomyces pombe: Synthesis from Ins(1,4,5)P3 and osmotic regulation. Biochem. J. 335, 671-679

40. Ives, E. B., Nichols, J., Wente, S. R., and York, J. D. (2000) Biochemical and functional characterization of inositol 1,3,4,5,6-pentakisphosphate 2-kinases. J. Biol. Chem. 275, 36575-36583

41. Dubois, E., Dewaste, V., Erneux, C., and Messenguy, F. (2000) Inositol polyphosphate kinase activity of Arg82/ArgRIII is not required for the regulation of the arginine metabolism in yeast. FEBS Lett. 486, 300-304.

42. Watson, P. J., Fairall, L., Santos, G. M., and Schwabe, J. W. (2012) Structure of HDAC3 bound to co-repressor and inositol tetraphosphate. Nature 481, 335-340 